\title{
Generation of Intense PEFs Using a Prolate Spheroidal Reflector Attached to the Bipolar Former of a 10-GW Pulsed Power Generator
}

\author{
Bucur M. Novac ${ }^{(0}$, Senior Member, IEEE, Renzhen Xiao ${ }^{(}$, Peter Senior, Member, IEEE, \\ Laurent Pécastaing ${ }^{\circledR}$, Senior Member, IEEE, and Ivor R. Smith, Senior Member, IEEE
}

\begin{abstract}
A prolate spheroidal reflector was designed, manufactured, and attached to a bipolar former at the output of a 10-GW-Tesla-driven Blumlein pulsed power generator. The reflector, operated in water, is capable of producing intense pulsed electric fields of the order of $50 \mathrm{kV} / \mathrm{cm}$. Constructional details are provided, together with experimental results, and a detailed analysis using 3-D software modeling of the reflector that provides results in good agreement with experimental data.
\end{abstract}

Index Terms-Antenna, pulse compression circuits, pulse modulation, pulsed electric fields (PEFs), pulsed power systems.

\section{INTRODUCTION}

A RANGE of modern pulsed power applications, including not only bioelectric studies and medical cancer treatment but also food industry processing and water sterilization, require the generation of intense pulsed electric fields (PEFs) in dielectric materials having a large value of permittivity.

Related to medical applications and using invasive electrode-based techniques, a number of research groups worldwide have successfully demonstrated that picosecond (ps) PEFs can induce apoptosis in cancer cells [1]-[4].

The use of ultrashort pulses with a rise time of the order of hundreds of ps provides also an opportunity of using noninvasive antenna techniques, instead of invasive electrodes, to deliver ps PEF to targets inside bodies. There are basically two possible configurations, both based on Carl Baum designs and pioneered at Old Dominion University, USA. In the first arrangement, a focusing antenna is placed in air and the very high electric fields are generated inside the biomedical target

Manuscript received December 4, 2017; revised February 27, 2018; accepted May 30, 2018. The review of this paper was arranged by Senior Editor W. Jiang. (Corresponding author: Bucur M. Novac.)

B. M. Novac, P. Senior, and I. R. Smith are with the Wolfson School of Mechanical, Electrical and Manufacturing Engineering, Loughborough University, Loughborough LE11 3TU, U.K. (e-mail: b.m.novac@ lboro.ac.uk).

R. Xiao is with the Northwest Institute of Nuclear Technology, Xi'an 710024, China (e-mail: xiaorenzhen@tsinghua.org.cn).

L. Pécastaing is with the Laboratoire des Sciences de l'Ingenieur Appliquees a la Mecanique et au Genie Electriques-Federation IPPRA, Université de Pau et des Pays de l'Adour, EA4581, 64000 Pau, France (e-mail: laurent.pecastaing@univ-pau.fr).

Color versions of one or more of the figures in this paper are available online at http://ieeexplore.ieee.org.

Digital Object Identifier 10.1109/TPS.2018.2844160 with the aid of a complex adapting impedance system made from a large number of lenses [5]. In the second arrangement, a prolate spheroidal reflector (PSR) is used and immersed in water to directly focus ps PEF inside a small volume of a target also placed in water [6].

This paper, representing the third phase of the Loughborough program aiming at developing noninvasive PEF systems, is using the second Baum arrangement. In the first phase, a 10-GW generator was built and tested [7], while in the second phase, a bipolar former (BF) was demonstrated to modulate and condition the generator voltage output to allow the efficient coupling to an antenna [8].

This paper presents the design, manufacture, and testing of a PSR. Experimental results are compared with predictions made using Computer Simulation Technology (CST) [9] 3-D modeling, before concluding remarks that include a brief comment on the next steps toward the development of a powerful ps PEF generator for medical applications.

\section{Design, Manufacture, And Modeling of THE PROLATE SPHEROIDAL REFLECTOR}

\section{A. Design and Manufacture}

The design of the PSR followed the technique described in [6] and the result is presented in Fig. 1. The PSR is immersed in water and coupled to the $50-\Omega$ coaxial transmission line output of the existing BF [8]. Using a formula presented in [6], the characteristic input impedance of the PSR can be calculated as

$$
Z=\frac{1}{\pi} \sqrt{\frac{\mu_{0}}{\varepsilon_{0} \varepsilon_{r}}} \ln \left(\cot \left(\frac{\theta_{0}}{2}\right)\right)
$$

where $\varepsilon_{r}=2.4$, since, in the present arrangement, polyethylene fills the space between the reflector and the conical wave launcher (CWL) and $\theta_{0}$ is its half-angle (see Fig. 1). For the design in Fig. $1, \theta_{0} \approx 21.3^{\circ}$ and therefore the input impedance of the PSR is $Z \approx 130 \Omega$. No efforts have been attempted at this stage to reduce the mismatch between the BF coaxial output and the PSR.

Manufacture of the PSR required a wooden frame to be built [Fig. 2(a)], after which 2-mm stainless steel sheet was used to obtain the required shape [Fig. 2(b)]. 


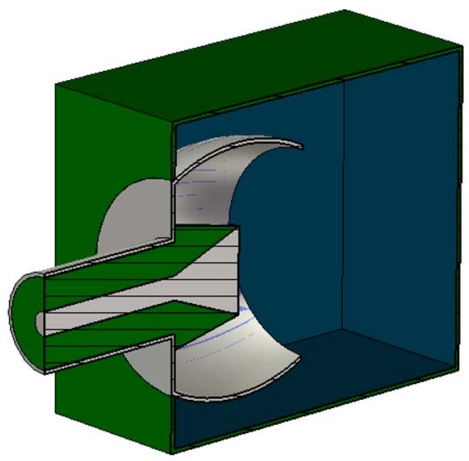

a)

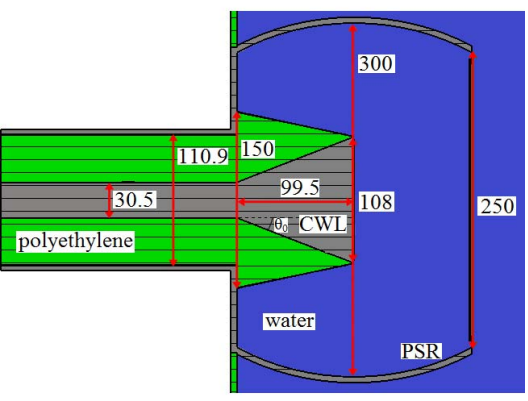

b)

Fig. 1. CST model drawings of the PSR immersed in water and attached to the $50-\Omega$ coaxial transmission line representing the BF output. (a) 3-D overview. (b) Details of the PSR with the corresponding CWL having a half-angle $\theta_{0}$. Dimensions are indicated in $\mathrm{mm}$.

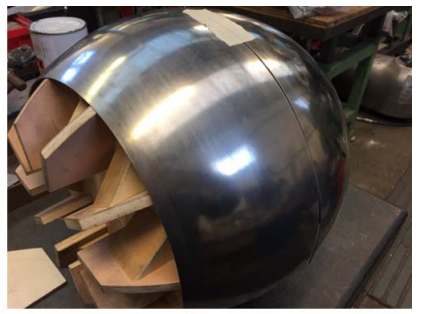

a)

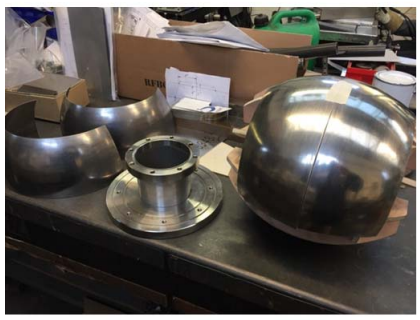

b)

Fig. 2. Manufacture of the PSR. (a) Reflector was manufactured with the help of a wooden frame. (b) Parts ready to be welded together.

\section{B. Modeling}

The electric field distribution generated by the PSR when energized by the BF was modeled in time domain using CST software. Results are presented in the following when compared with the experimental data.

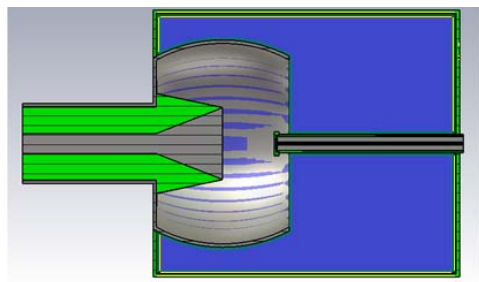

a)

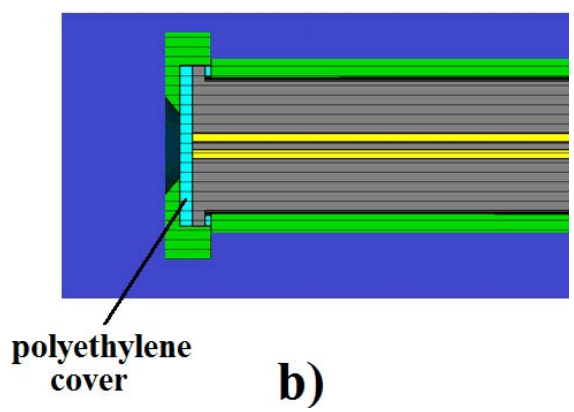

Fig. 3. D-dot probe modeled with CST. (a) D-dot probe assembly; the probe is made from an N-type jack with the pin cut flush with the flange and covered with 2-mm-thick polyethylene sheet, as indicated; after the probe is connected to a coaxial cable, the assembly is housed in a plastic tube. (b) Overview showing the D-dot probe assembly mounted on the PSR axis, $79 \mathrm{~mm}$ away from the CWL.

\section{Diagnostic Tools}

The results obtained during the tests have been obtained using two differentiating sensors: a V-dot probe [10] mounted on the BF coaxial output at $390 \mathrm{~mm}$ from the PSR and a D-dot probe mounted in water on the PSR axis at $79 \mathrm{~mm}$ from the CWL, as shown in Fig. 3. The V-dot probe is described in [8] and is made from an $18-\mathrm{GHz} \mathrm{N}$-type female-female adaptor, without any modifications being required. The D-dot probe is made from an N-type straight jack connector with the pin cut flush with the metallic flange and covered with a piece of 2-mm-thick polyethylene sheet (Fig. 3). The polyethylene cover is required to lower the probe output voltage to less than $0.5 \mathrm{kV}$. After connected to the coaxial cable, the probe is mounted inside a plastic tube and positioned along the PSR axis. Both differentiating probes have been modeled using CST software, with predicted calibration factors very close to those obtained in the real tests.

The probes are both connected to $18-\mathrm{GHz}$ coaxial cables and attenuators, with signals recorded using an $18-\mathrm{GHz}$, 60-GS/s digital oscilloscope. As the peak voltage generated by the V-dot probe reaches only a few tens of volts, a "standard" $20-\mathrm{dB}$ attenuator is used, capable of withstanding a voltage peak of $100 \mathrm{~V}$. The peak voltage generated by the D-dot probe is, however, in excess of $400 \mathrm{~V}$ and required use of a 20-dB, 30-GHz Barth Electronics [11] Model 142 series attenuator, capable of withstanding $2.5-\mathrm{kV}$ input and which is further coupled to a chain of two "standard" 20- and 10-dB attenuators.

\section{Water}

As shown in Fig. 4, the PSR is mounted inside a plastic cube of $0.5-\mathrm{m}$ side, which is filled with demineralized water. 


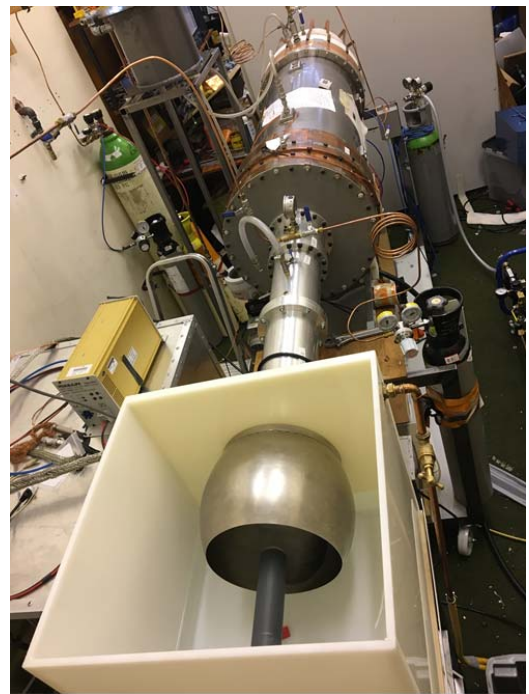

Fig. 4. Overview of the experimental arrangement, with the PSR mounted inside a plastic cube and the D-dot probe assembly mounted on its axis. During testing, the cube is filled with water.
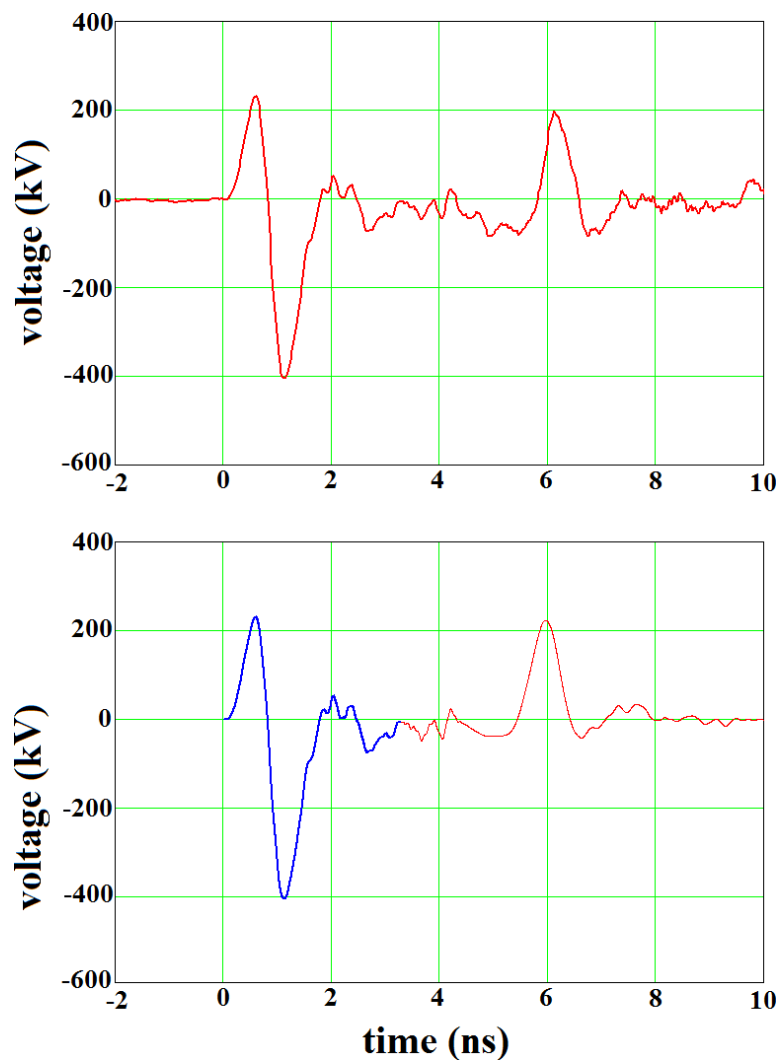

Fig. 5. Time variation of the voltage impulse in the $50-\Omega$ coaxial transmission line connecting the BF with the PSR. Top: experimental data; bipolar voltage impulse measured by the V-dot attached to the coaxial transmission line. Bottom: CST data; the first 3 ns of the experimental voltage signal (highlighted) are used as CST input data. The rest of the voltage signal, including the voltage reflection (around $6 \mathrm{~ns}$ ) due to coaxial line-PSR impedance mismatch, is predicted by CST.

The water can be continuously filtered in order to maintain a resistivity of $20 \mathrm{M} \Omega \cdot \mathrm{cm}$.

For modeling the propagation of electromagnetic waves through water, a Debye model which takes into account the losses was readily available in the CST library.
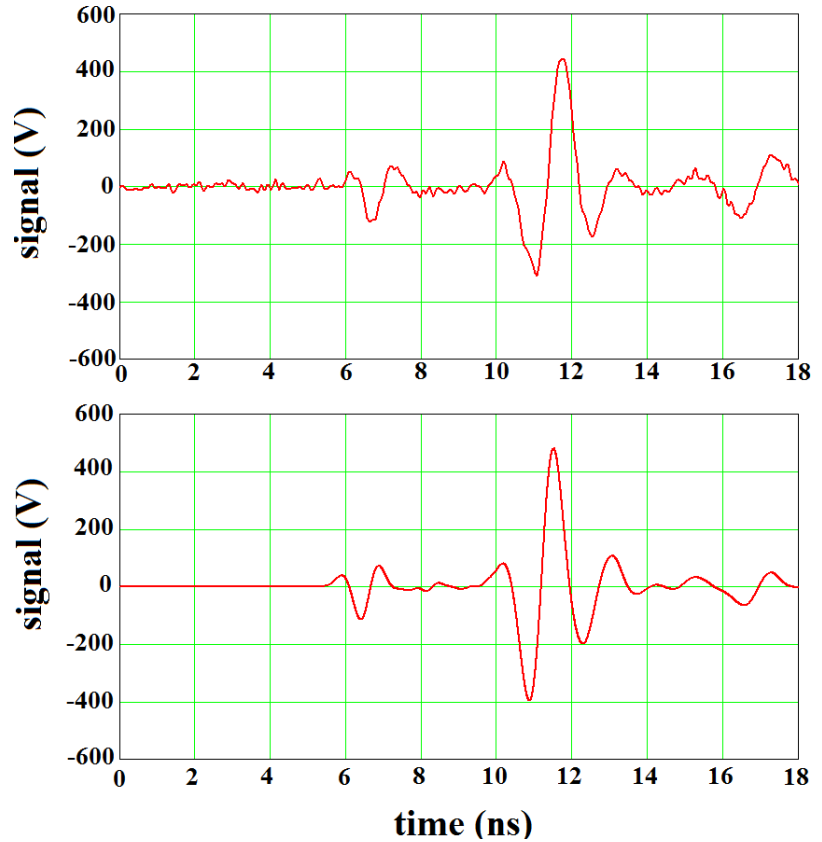

Fig. 6. Typical D-dot signal during a test (time origin as in Fig. 5). Top: experimental data; rough signal, as recorded. Bottom: CST predicted data.
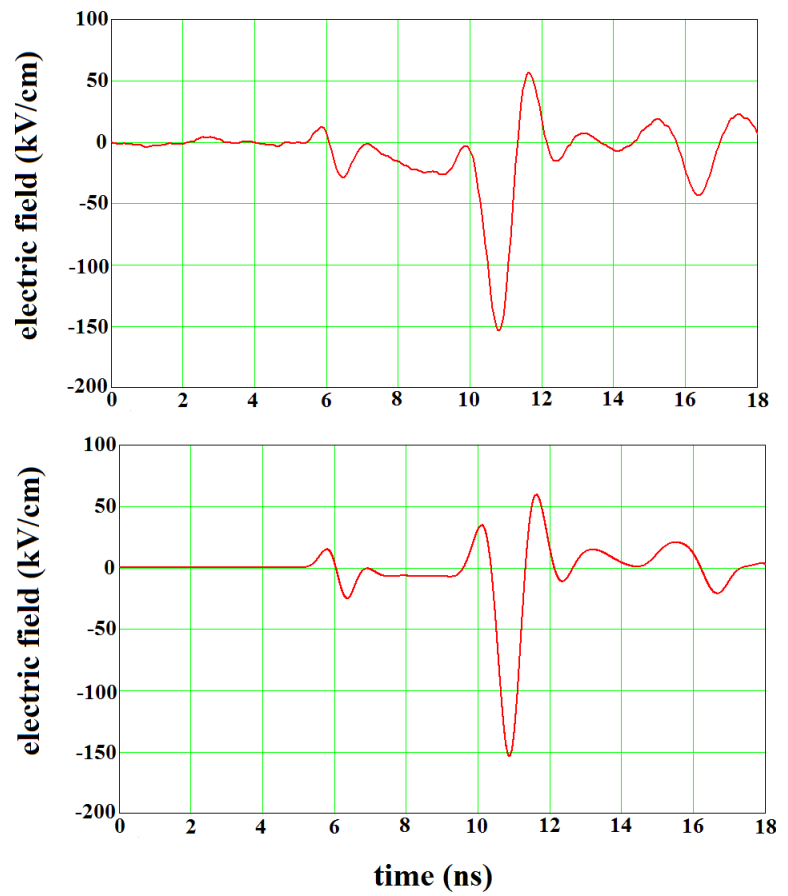

Fig. 7. Time variation of the electric field strength in water, near the D-dot probe polyethylene cover (time origin as in Fig. 5). Top: integrated experimental data.Bottom: CST predicted data.

\section{EXPERIMENTAL RESUlts COMPARED With CST SOFTWARE PREDICTIONS}

The main results obtained during the experimental campaign are presented in Figs. 5-7. The PSR is energized by the voltage impulse generated by BF shown in Fig. 5, with a (negative) time rate-of-change peak close to $2.5 \mathrm{MV} / \mathrm{ns}$. Data from the first $3 \mathrm{~ns}$ of the voltage impulse, obtained by integrating the 


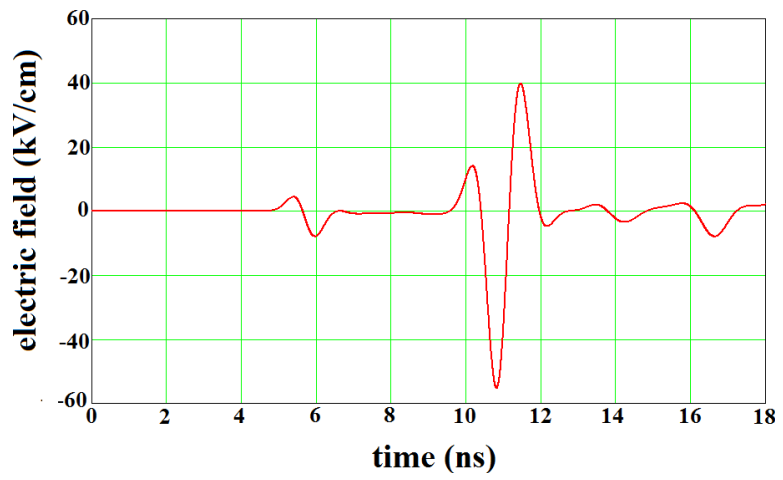

Fig. 8. CST predicted time variation of the electric field strength in water in the (second) PSR focus point, in the absence of the D-dot probe assembly (time origin as in Fig. 5).
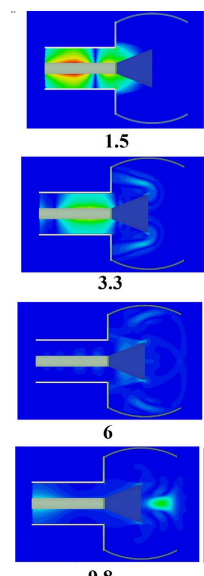

9.8
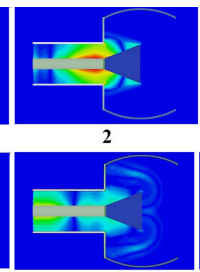

3.8
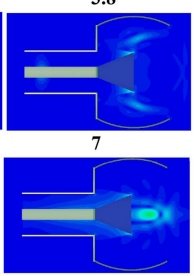

10.4
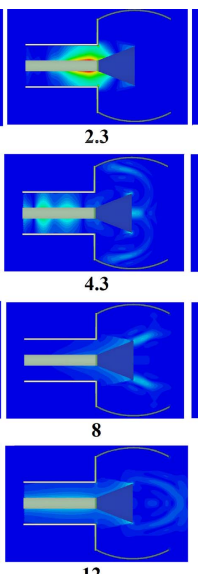

12
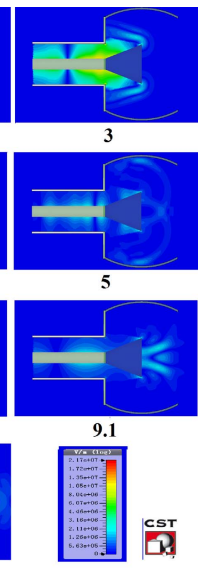

Fig. 9. CST predicted time variation of the electric field distribution in water and polyethylene, corresponding to Fig. 8 (stills from a movie). Numbers indicate the time in ns, with time origin as in Fig. 5.

time rate-of-change signal recorded from the V-dot probe, are used as input for the CST model. Due to the impedance mismatch between the coaxial line and the PSR, a reflected voltage impulse can be observed at about $6 \mathrm{~ns}$, both on CST predictions and on the experimental data (Fig. 5).

The time rate-of-change signal recorded from the D-dot probe is shown in Fig. 6 and compared with the CST prediction. The integrated data, representing the electric field in water near the D-dot polyethylene cover, are presented in Fig. 7. A good agreement can be noticed between the experimental data and the CST calculations. This enables the CST to be used to predict the electric field, presented in Fig. 8, which is generated in the absence of the perturbing D-dot assembly in a $\mathrm{cm}^{3}$ size spot surrounding the PSR (second) focal point [6]. The corresponding time variation of the electric field distribution is shown in Fig. 9, where one can distinguish the three types of waves described in [6]: prepulse at $5 \mathrm{~ns}$, the impulse at $10.4 \mathrm{~ns}$, and the superposition of the first two.

\section{CONCLUSION}

A PSR was designed, manufactured, modeled, and attached to the $\mathrm{BF}$ of a $10-\mathrm{GW}$ pulsed power generator, and was tested immersed in water. The results obtained indicate the possibility to generate in water the peak electric fields in the order of $50 \mathrm{kV} / \mathrm{cm}$.

The results open the way for interesting biomedical proofof-principle experiments, allowing the system to be used in the near future for preliminary in vitro and in vivo testing.

In the next phase of the program, it is intended to develop a much more compact and efficient PEF system, capable of operating at a high repetition rate.

\section{REFERENCES}

[1] K. H. Schoenbach et al., "The effect of intense subnanosecond electrical pulses on biological cells," IEEE Trans. Plasma Sci., vol. 36, no. 2, pp. 414-422, Apr. 2008.

[2] S. Xiao, S. Guo, V. Nesin, R. Heller, and K. H. Schoenbach, "Subnanosecond electric pulses cause membrane permeabilization and cell death," IEEE Trans. Biomed. Eng., vol. 58, no. 5, pp. 1239-1245, May 2011.

[3] Y. Y. Hua, X. S. Wang, Y. Zhang, C. G. Yao, X. M. Zhang, and Z. A. Xiong, "Intense picosecond pulsed electric fields induce apoptosis through a mitochondrial-mediated pathway in HeLa cells," Mol. Med. Rep., vol. 5, no. 4, pp. 981-987, 2012.

[4] J. Jia, Z. A. Xiong, Q. Qin, C. C. Yao, and X. Z. Zhao, "Picosecond pulsed electric fields induce apoptosis in a cervical cancer xenograft," Mol. Med. Rep., vol. 11, no. 3, pp. 1623-1628, 2015.

[5] P. Kumar et al., "A hyperband antenna to launch and focus fast highvoltage pulses onto biological targets," IEEE Trans. Microw. Theory Techn., vol. 59, no. 4, pp. 1090-1101, Apr. 2011.

[6] S. Xiao, M. A. Migliaccio, J. C. Camp, J. F. Kolb, K. H. Schoenbach, and C. E. Baum, "A wide-band focusing system for bioelectric applications," in Proc. 16th IEEE Int. Pulsed Power Conf., vol. 1, Jun. 2007, pp. 744-747.

[7] B. M. Novac, M. Wang, I. R. Smith, and P. Senior, "A 10 GW Tesladriven Blumlein pulsed power generator," IEEE Trans. Plasma Sci., vol. 42, no. 10, pp. 2876-2885, Oct. 2014.

[8] M. Wang, B. M. Novac, L. Pécastaing, and I. R. Smith, "Bipolar modulation of the output of a $10-\mathrm{GW}$ pulsed power generator," IEEE Trans. Plasma Sci., vol. 44, no. 10, pp. 1971-1977, Oct. 2016.

[9] Computer Simulation Technology. Accessed: Nov. 2017. [Online]. Available: https://www.cst.com/Products

[10] C. A. Ekdahl, "Voltage and current sensors for a high-density z-pinch experiment," Rev. Sci. Instrum., vol. 51, no. 12, pp. 1645-1648, 1980.

[11] Barth Electronics Inc. Accessed: Nov. 2017. [Online]. Available: https://www.barthelectronics.com

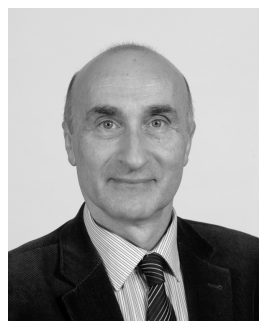

Bucur M. Novac (M'06-SM'08) received the M.Sc. and Ph.D. degrees from the University of Bucharest, Bucharest, Romania, in 1977 and 1989, respectively. In 1998. He joined Loughborough University, Loughborough, U.K., where he is currently a Professor of pulsed power. He has co-authored 2 books on explosive pulsed power and has authored over 200 refereed papers and conference contributions. His current research interests include compact and repetitive high-power systems, explosively and electromagnetically driven magnetic flux compression generators and their applications, electromagnetic launchers, ultrafast magneto and electrooptic sensors, and 2-D modeling of pulsed power systems.

Prof. Novac is a Voting Member of the Pulsed Power Science \& Technology Committee of the IEEE Nuclear and Plasma Science Society. He is also a member of the International Steering Committees for the MEGAGAUSS Conferences and the Euro-Asian Pulsed Power Conferences. He is also a member of the Organizing Committee for the IEEE International Power Modulator and High Voltage Conference and a Co-Chairman of the U.K. Pulsed Power Symposium. He is a Chartered Engineer and a fellow of the Institution of Engineering and Technology, U.K. 


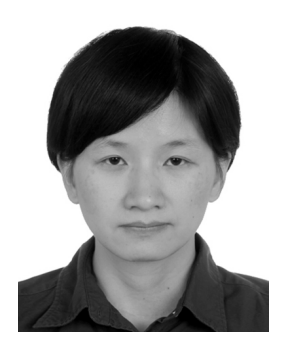

Renzhen Xiao received the Ph.D. degree in nuclear science and technology from Tsinghua University, Beijing, China, in 2007.

Since 2002, she has been with the Northwest Institute of Nuclear Technology, Xi'an, China. Her current research interests include relativistic electronics and high-power microwave generation and transmission.

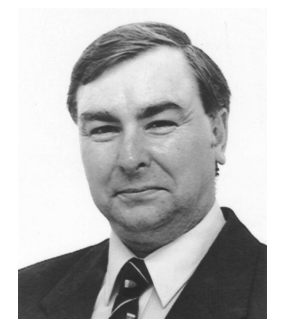

Peter Senior (M'16) received the Honours degree in physics with electronics from Leicester University, Leicester, U.K.

$\mathrm{He}$ was with the Electronic and Electrical Engineering Department, Loughborough University, Loughborough, U.K., where he was involved in ultrasonic nondestructive evaluation, extending into nonlinear (finite amplitude) acoustics. He was one of the founder members of the Pulsed Power Research Group, Loughborough University. He was involved in propellant and explosive pulsed MHD generators, and also produced systems to support a flux compressor program, highefficiency launchers, and the electromagnetic protection of armoured vehicles. He has produced a number of transportable high-energy high-power systems, varying in size between fitting into a van, and up to two ISO containers, supplied by diesel generators. This has required expertise in the health and safety aspects of integrating pulsed power systems with the equipment of sponsoring and collaborating bodies.

Mr. Senior is a member of the Institute of Physics, London, U.K., and a Chartered Physicist.

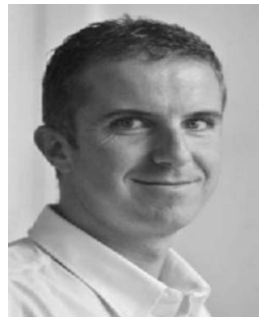

Laurent Pécastaing (M'13-SM'17) received the Ph.D. degree and the Research Directorship Habilitation in electrical engineering from the Université de Pau et des Pays de l'Adour (UPPA), Pau, France, in 2001 and 2010, respectively.

Since 2016, he has been a Full Professor with the Laboratoire SIAME, UPPA. He is the Head of the Electrical Engineering Team with the Laboratoire SIAME and he is also the Director of a Common Laboratory between Le Commissariat à l'Energie Atomique, Avon-les-Roches, France, and UPPA. His current research interests include high-power microwave sources, compact pulsed power devices, and ultrafast transient probes.

Dr. Pécastaing is the Chairman of the next Euro-Asian Pulsed Power Conference-BEAMS Conference to be held in France in 2020.

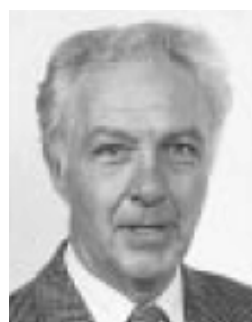

Ivor R. Smith (M'05-SM'11) received the B.Sc., Ph.D., and D.Sc. degrees from the University of Bristol, Bristol, U.K.

He completed an indentured student apprenticeship with Witton Works of the General Electric Company, Boston, MA, USA. He then became a Lecturer with the University of Birmingham, Birmingham, U.K., subsequently becoming a Senior Lecturer and a Reader. He was a Professor of electrical power engineering with Loughborough University, Loughborough, U.K., where he was the Head of the Department, the Dean of Engineering, and a Pro-Vice Chancellor. His current research interests include the production, conditioning, and utilization of large pulses of electrical energy and his work has brought in very substantial funding from a variety of sources.

Dr. Smith is a Chartered Engineer and a fellow of both the Institution of Engineering and Technology and the Royal Academy of Engineering. 